\title{
Optical Flow Based Block Matching Method for Tendon Tracking in Ultrasound
}

\author{
Bo-I Chuang ${ }^{\mathrm{a}}$, Jian-Han Hsu ${ }^{\mathrm{a}}$, Li-Chieh Kuo ${ }^{\mathrm{b}}$, Fong-Chin Su ${ }^{\mathrm{b}}$, I-Ming Jou ${ }^{\mathrm{c}}$, Yung-Nien Sun ${ }^{\mathrm{a}, *,+}$ \\ ${ }^{\mathrm{a}}$ Department of Computer Science and Information Engineering, National Cheng Kung University, No.1 University \\ Road, Tainan 701, Taiwan \\ ${ }^{\mathrm{b}}$ Department of Biomedical Engineering, National Cheng Kung University, No.1 University Road, Tainan 701, \\ Taiwan \\ ${ }^{\mathrm{c}}$ Department of Orthopedics, National Cheng Kung University Hospital, No.138 Sheng Li Road, Tainan 704, Taiwan
}

*Corresponding Author: ynsun@mail.ncku.edu.tw

\begin{abstract}
Tendon motion is one of the important features used in tendinopathy diagnosis. Ultrasound image is commonly used to observe the tendon motion. However, the speckle noise and out-of-plane issues cause the tracking process difficult. Manual tracking requires lots of time and will obtain different results between users. In order to track the tendon motion automatically, we develop a tracking method combining the advantages of optical flow method and multi-kernel block matching for tracking motion of finger tendon in ultrasound images. The proposed method selects the frame interval for block matching adaptively and reduces the accumulated tracking error in block matching. For every adjacency frame, optical flow is computed and also used to estimate the accumulated displacement. Multi-kernel block matching is then applied to the two selected frames when the accumulated displacement between these two frames is large enough. In the experiments, the cadaver data are used to evaluate the tracking results. The average absolute error in cadaver data is less than $0.05 \mathrm{~mm}$. It also shows that the proposed method can track the motion of tendon in vivo which can provide the useful information for clinical diagnosis.
\end{abstract}

Keywords: tendon tracking, ultrasound, optical flow, block matching.

+ This work was supported by MOST, Taiwan under grant MOST 104-2221-E-006-097-MY3

\section{Introduction}

Tendon is a band of fiber connective tissue connecting muscle to bone. Tendon motion is an important feature to evaluate the normality of the limbs and joints functions. In clinical application, ultrasound is usually used to observe the tendon motion. Some researches report that tendon behavior will change if tendinopathy occur ${ }^{(1)}$.

In this research, flexor tendon in finger is tracked. The tendinopathy of flexor tendon will cause trigger finger, which symptom is occurred by repeatedly and incorrectly forcing. Figure 1 shows the anatomical structure of finger in sagittal view of ultrasound image at first annular pulley (A1 pulley). Flexor tendon in finger including flexor digitorum superficialis (FDS) and flexor digitorum profundus (FDP) tendons have different motions when finger flexion and extension at different poses, therefore two tendons cannot be tracked as one moving target. Although the tendon motion can be observed in ultrasound sequence, the speckle noise in the ultrasound image sequence makes the tendon tracking process difficult. Furthermore, if the acquisition direction is not parallel to the direction of motion, out-of-plane issue will also occur. This issue causes the texture of tissue changed during motion and leads to the instability of tissue tracking.

Many researching groups develop different tracking methods for ultrasound images. Optical flow and block matching are the two commonly used methods for tissue tracking. In many literatures, these two methods have been modified to improve the accuracy and performance for different types of tissue tracking ${ }^{(2-8)}$. Although block 
matching and optical flow method can track the moving target in ultrasound images, there are still several challenges need to be resolved. First, the cumulative error of block matching method will increase over time. The cumulative error occurs because the tracking error is added up when the reference template updates with inaccurate displacement. Second, the displacement cannot be too large while applying optical flow method. Due to the intensity consistency constraint, the optical flow method will underestimate the displacement if the motion is too large. At last, the intervals of tracking frames need to be decided. Though many methods used the adjacency frame or constant frame interval in block matching or optical flow method, dynamic frame interval will effectively increase the tracking accuracy.

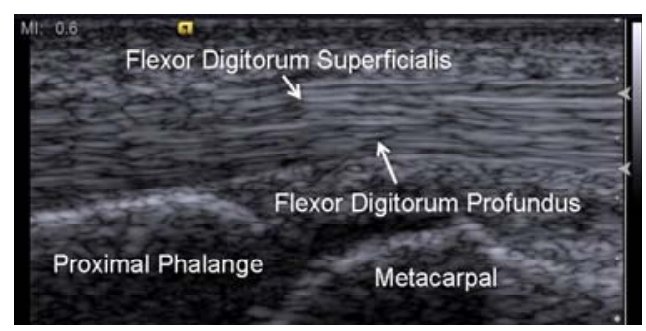

Fig. 1. Finger ultrasound image in sagittal view.

In this research, we have developed an automatic tracking method combining block matching and optical flow methods. The proposed method designs a suitable index for determining the frame interval adaptively. In the experiments, cadaver data are used to validate the proposed method. Furthermore, optical flow and block matching methods are also used to compare the tracking accuracy with the proposed method.

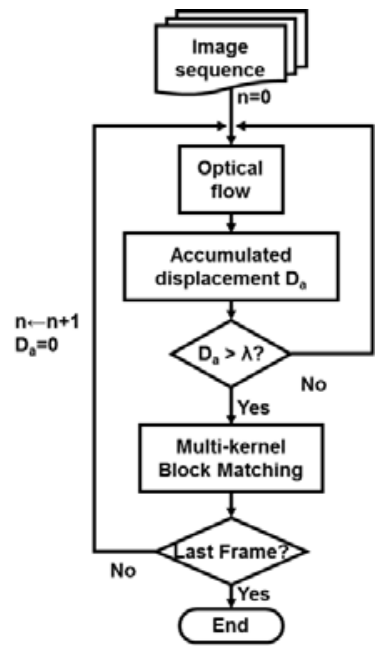

Fig. 2. Flowchart of proposed method.

\section{Method}

\subsection{Overview of Proposed Method}

Figure 2 shows the flowchart of proposed method. The optical flow is first used to compute the displacement in adjacency frames. The multi-kernel block matching (MKBM) is then applied in the two selected frames which are the starting frame and the frame whose accumulated displacement is larger than a given constant $\lambda$.

\subsection{Optical Flow Method}

Optical flow is a classical tracking method. Lucas and Kanade $^{(9)}$ presented a differential method for optical flow estimation. The displacement of a point can be obtained as the least square solution with the following optical flow equation using:

$$
\left[\begin{array}{cc}
I_{x}\left(p_{0}\right) & I_{y}\left(p_{0}\right) \\
I_{x}\left(p_{1}\right) & I_{y}\left(p_{1}\right) \\
\vdots & \vdots \\
I_{x}\left(p_{n}\right) & I_{y}\left(p_{n}\right)
\end{array}\right]\left[\begin{array}{l}
V_{x} \\
V_{y}
\end{array}\right]=-\left[\begin{array}{c}
I_{t}\left(p_{1}\right) \\
I_{t}\left(p_{2}\right) \\
\vdots \\
I_{t}\left(p_{n}\right)
\end{array}\right],
$$

where $\mathrm{p}_{\mathrm{n}}$ is the $\mathrm{n}$-th neighbor point in the computing block. $I_{x}, I_{y}$, and $I_{t}$ are the derivatives of the pixel at $(x, y, t)$ in the $\mathrm{x}, \mathrm{y}$, and time directions. $\mathrm{V}_{\mathrm{x}}$ and $\mathrm{V}_{\mathrm{y}}$ are the $\mathrm{x}$ and $\mathrm{y}$ components of velocity at position $(x, y)$.

In the implementation of optical flow method, we select a rectangle region as the tracking target in the first frame. To compute the block motion, instead of averaging the displacements of all pixels in the block, we exclude the outlier pixels whose moving direction is different to the main moving direction. Only the remaining pixels are used to compute the overall displacement of tracking target. The displacement is then added up to check whether the accumulated displacement is large enough.

\subsection{Multi-kernel Block Matching}

MKBM is the modified block matching scheme proposed by Korstanje et al. ${ }^{(4)}$. In block matching, the block size is an important factor influencing the motion tracking. If the block size is too small, the information is not enough, leading to ambiguous tracking results. On the contrary, if the block size is too large, the local deformation may be smoothed out and lead to underdetermined estimations. MKBM separates the reference block into several sub-blocks. Each sub-block is at first applied with block matching method to find the best matching block. The 
matching results of all sub-blocks are then combined to obtain the overall matching result.

In our implementation of MKBM method, we separate the target region into four sub-blocks with ten pixels overlapped. The similarity of each sub-block is computed using the sum of absolute difference (SAD):

$$
S A D=\frac{1}{M N} \sum_{j=1}^{N} \sum_{i=1}^{M}\left|T_{i, j}-R_{i, j}\right|
$$

where $\mathrm{M}$ and $\mathrm{N}$ are the width and height of sub-block, and $T_{i, j}$ and $R_{i, j}$ are the intensity values of pixel (i, j) at the target region and reference template. Considering the soft tissue adjacent to the tendon may passively move with smaller displacement, we compute the block displacement by choosing the maximal value of the four sub-blocks:

$\mathrm{D}_{\mathrm{t}}=\operatorname{Max}\left(\mathrm{D}_{\mathrm{t}, 1}, \mathrm{D}_{\mathrm{t}, 2}, \mathrm{D}_{\mathrm{t}, 3}, \mathrm{D}_{\mathrm{t}, 4}\right)$,

where $D_{t, n}$ is the displacement of $n$-th sub-block at $t$-th frame. The displacement between the two selected frames can then be obtained by using linear interpolation.

\section{Experiment Results}

\subsection{Data Acquisition}

The ACUSON S2000 Ultrasound System (Siemens Medical Solutions, Mountain View, CA, USA) with 14 $\mathrm{MHz}$ transducer is used to acquire the ultrasound images. The acquired video is $1024 \times 768$ in image size with pixel resolution $0.0265 \mathrm{~mm} / \mathrm{pixel}$, and the frame rate is $30 \mathrm{fps}$. The subjects laid their right arm on the table with palm facing up with finger flexion pose. Subjects are asked to virtually push a hard plate which is not moved in fact by flexing their PIP or DIP joints. Ultrasound probe is placed above the A1 pulley region parallel to the tendon direction.

In order to evaluate the accuracy of proposed method, finger tendon image acquired from cadaver are also used. To simulate the finger motion in cadaver, we press the cadaver's finger which is then released by the weights hanged on the tendon with string at the elbow side as shown in figure 3. A tiny iron plate is inserted in FDS tendon closed to A1 pulley as a marker. The ultrasound probe is placed above the region covering the A1 pulley and marker. The acquired cadaver ultrasound image is shown in figure 4.

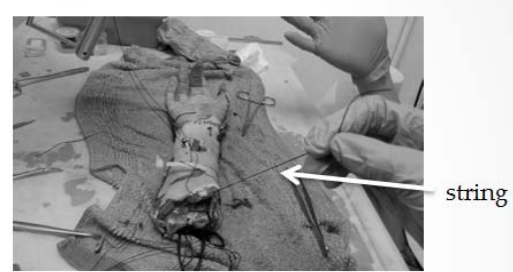

Fig. 3. Cadaver with string tied to the tendon.

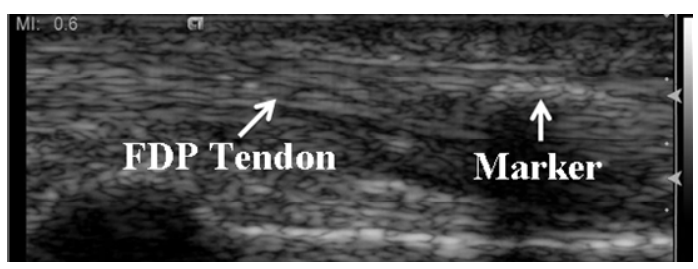

Fig. 4. Finger ultrasound image of cadaver.

\subsection{Tracking Results of Proposed Method}

Figure 5 is the tracking result of the proposed algorithm in finger ultrasound. Figure 5(a) is the tracking target of FDP tendon at the first frame. Figure 5(b) and (c) are the tracking results at frame 150 and 300 in finger motion. Although FDP tendon is attached on the distal phalange (DP), the movement of tendon induced by DP is larger than the one of the underlying bone. Figure 5(b) and (c) show that the tracking results agree with this statement.

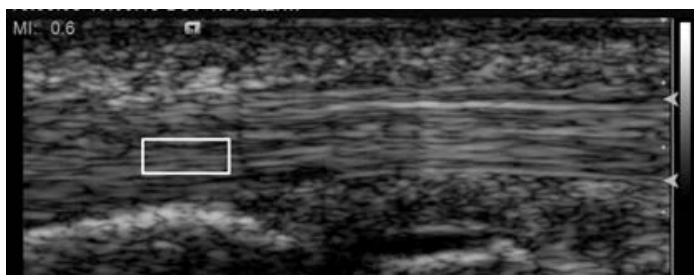

(a)

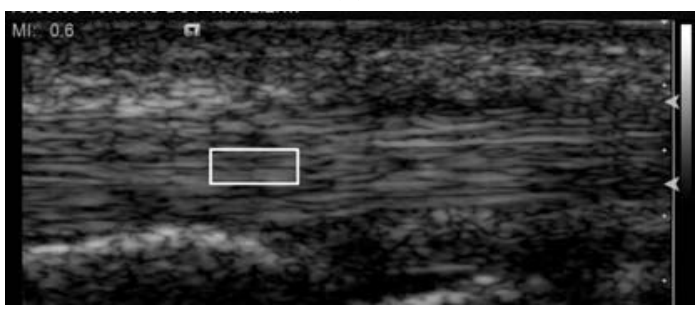

(b)

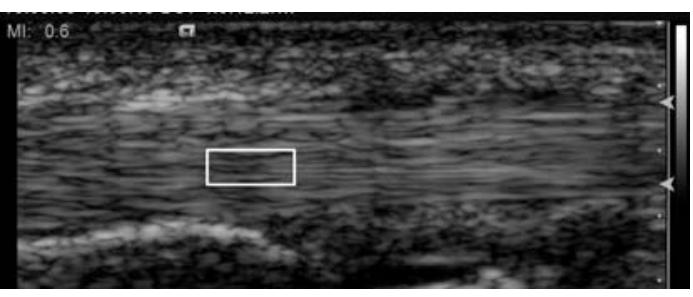

(c)

Fig. 5. Tracking results of proposed method in FDP tendon.

(a) Initial frame; (b) frame 150; (c) frame 300. 


\subsection{Validation}

To validate the tracking results of tendon motions from cadaver, the motions of marker inside the FDP tendon is treated as the ground truth of tendon motion. The position of marker can be easily tracked manually due to its stable appearance during tendon sliding. As the tracking region and the marker are located in the same tendon, the distance between tracking region and marker should be stable during the tracking process. As shown in figure 6, the results of proposed method are indicated as white block, and the ground truths are indicated as white circle. The distance between tracking target and ground truth are stable in all frames. It shows that the tracking results match the ground truth throughout the tracking process.
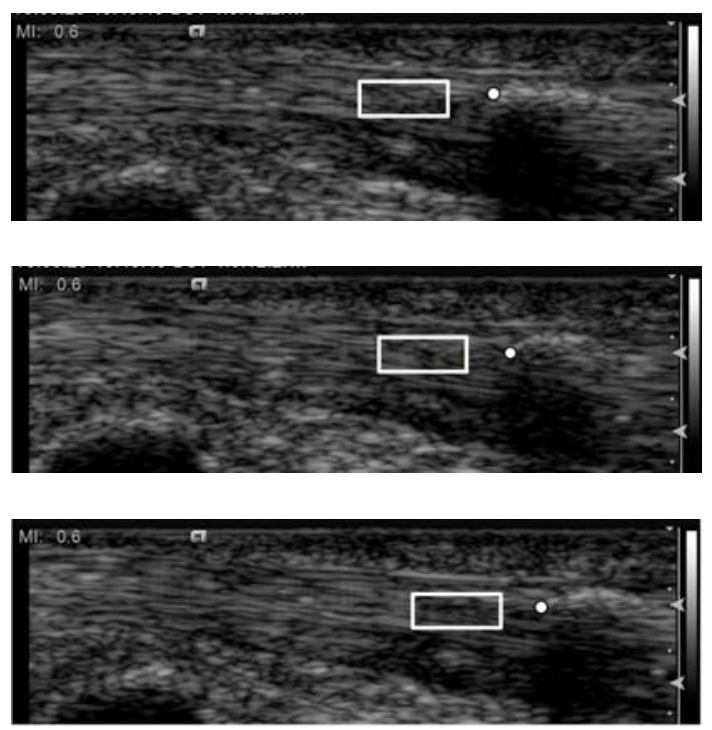

Fig. 6. Tracking results and the ground truths in cadaver data. (a) Initial frame; (b) frame 150; (c) frame 300.

To quantify the tracking difference between the ground truth and the proposed method, two metrics, relative error $E_{r}$, and average absolute error $E_{a}$, are used.

$E_{r}=\left|\frac{D_{G T}-D_{P A}}{D_{G T}}\right| * 100 \%$,

$E_{a}=\sum_{1}^{N} \frac{\left|d_{G T}-d_{P A}\right|}{N}$

where $D_{G T}$ and $D_{P A}$ are the total displacements given by ground truth and proposed method, $d_{G T}$ and $d_{P A}$ are the instantaneous displacements at t-th frame given by ground truth and proposed method, and $\mathrm{N}$ is the total number of frames. These two metrics are calculated for each motion case as shown in Table 1 . The results show the high accuracy achieved by the proposed method.

Table 1. Quantitative evaluation of proposed method.

\begin{tabular}{|c|c|c|c|c|c|}
\hline Case & 1 & 2 & 3 & 4 & 5 \\
\hline$E_{\mathrm{r}}(\%)$ & 0.65 & 2.52 & 2.08 & 3.33 & 0.96 \\
\hline$E_{\mathrm{a}}(\mathrm{mm})$ & 0.01 & 0.06 & 0.01 & 0.04 & 0.02 \\
\hline
\end{tabular}

\subsection{Comparison with Other Methods}

To compare the proposed method with other well-known methods, the optical flow and MKBM methods are applied. Figure 7 depicts the ground truths and the tracking results of optical flow, MKBM, and proposed method in three different cases. Both optical flow method and MKBM cannot track the tendon well in the whole motion sequence. The results of proposed method are closer to the ground truth in all cases. It is worth to mention that in figure $7(\mathrm{c})$, our method has large difference in displacement between frames 130 and 270 comparing to the ground truth. The major reason is that the linear interpolation is applied for the detailed displacement in between the two frames after MKBM. The interpolated result suffers large deviation from the ground truth when motion direction changes. It can be resolved by using other interpolation methods.

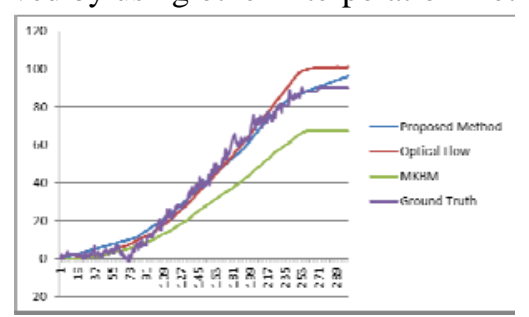

(a)

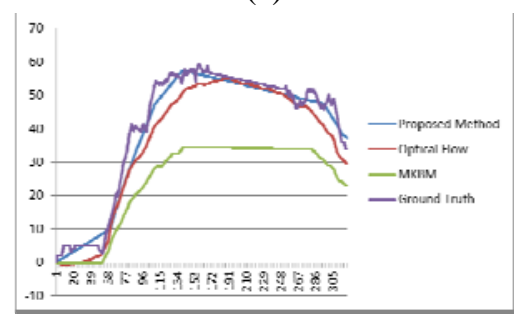

(b)

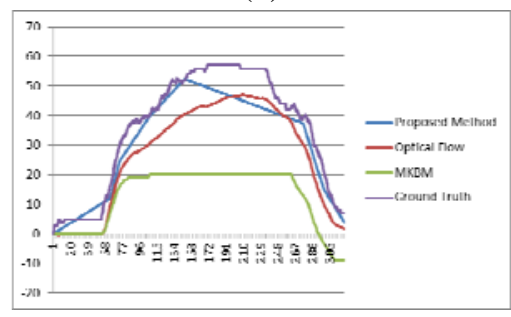

(c)

Fig. 7. The tendon tracking results of different tracking methods. 


\section{Conclusions}

In this paper, we propose a new tracking method for tendon tracking in ultrasound. In the proposed method, we select the frame interval adaptively based on the accumulated displacement computed by optical flow method. MKBM is then applied with the selected frame interval to obtain the better tracking results. In the experiments, the proposed method can correctly track the tendon motion in cadaver data, and has better tracking results comparing with the optical flow and MKBM methods. In the future, the interpolation process after applying MKBM can be further improved to obtain the more accurate displacement. We will also acquire more data to evaluate the accuracy and performance of proposed method. Furthermore, other tendons, such as Achilles tendon or common extensor tendon, can also be acquired to evaluate the feasibility of proposed method in different tissues.

\section{Acknowledgment}

The authors would like to express their gratitude for the grant under contract MOST 104-2221-E-006-097-MY3 from the Ministry of Science and Technology, Taiwan, R.O.C..

\section{References}

(1) Andrea S. Klauser, Ralph Faschingbauer, and Werner R. Jaschke. "Is sonoelastography of value in assessing tendons?" Seminars in musculoskeletal radiology. Vol. 14. No. 3. 2010.

(2) Guillaume Zahnd, et al. "Evaluation of a Kalman-based block matching method to assess the bi-dimensional motion of the carotid artery wall in B-mode ultrasound sequences." Medical image analysis 17.5 (2013): 573-585.

(3) Ryo Matsuzawa, et al. "Monitoring of lesion induced by high-intensity focused ultrasound using correlation method based on block matching." Japanese Journal of Applied Physics 51.7S (2012): 07GF26.

(4) Jan-Wiebe H. Korstanje, et al. "Development and validation of ultrasound speckle tracking to quantify tendon displacement." Journal of biomechanics 43.7 (2010): 1373-1379.

(5) Alia Siddig, Razaz K. Yousif, and Muhanned Alanwer. "Motion estimation in ultrasound image using dynamic multi-shape search." Computing, Electrical and Electronics Engineering (ICCEEE), 2013 International Conference on. IEEE, 2013.

(6) Elif Ayvali, and Jaydev P. Desai. "Optical Flow-Based Tracking of Needles and Needle-Tip Localization Using Circular Hough Transform in Ultrasound Images." Annals of biomedical engineering 43.8 (2015): 1828-1840.

(7) Daniel Tenbrinck, et al. "Histogram-based optical flow for motion estimation in ultrasound imaging." Journal of mathematical imaging and vision 47.1-2 (2013): 138-150.

(8) Daniel Barbosa, et al. "Fast tracking of the left ventricle using global anatomical affine optical flow and local recursive block matching." Proceedings of MICCAI CETUS challenge (2014).

(9) Bruce D. Lucas, and Takeo Kanade. "An iterative image registration technique with an application to stereo vision." IJCAI. Vol. 81. 1981. 